\title{
Mode Locking in a Free-Electron Laser Amplifier
}

\author{
N. R. Thompson ${ }^{1,2, *}$ and B. W. J. McNeil ${ }^{1, \dagger}$ \\ ${ }^{1}$ University of Strathclyde (SUPA), Glasgow G4 ONG, United Kingdom \\ ${ }^{2} A S T e C$, Daresbury Laboratory, Warrington WA4 4AD, United Kingdom
}

(Received 26 February 2008; published 21 May 2008)

\begin{abstract}
A technique is proposed to generate attosecond pulse trains of radiation from a free-electron laser amplifier. The optics-free technique synthesizes a comb of longitudinal modes by applying a series of spatiotemporal shifts between the copropagating radiation and electron bunch in the free-electron laser. The modes may be phase locked by modulating the electron beam energy at the mode spacing frequency. Three-dimensional simulations demonstrate the generation of a train of 400 as pulses at gigawatt power levels evenly spaced by $2.5 \mathrm{fs}$ at a wavelength of $124 \AA$. In the x-ray at wavelength $1.5 \AA$, trains of 23 as pulses evenly spaced by 150 as and of peak power up to $6 \mathrm{GW}$ are predicted.
\end{abstract}

PACS numbers: 42.60.Fc, 41.60.Cr, 42.65.Re

Attosecond pulses of high power, short wavelength, electromagnetic radiation would enable observation and possible control of very fast phenomena at the atomic time scale [1]. A new source of high-power x-ray pulses is the high gain free-electron laser (FEL) [2]. However, in the self-amplified spontaneous emission (SASE) mode of operation [3], the radiation has limited temporal coherence [4] with widths approximately that of the electron bunch source, typically many tens of femtoseconds. Several techniques have already been identified that may shorten this time scale into the attosecond regime. Selection of a short harmonic spike in a multiple undulator harmonic cascade may be one option [5]. Other techniques premodulate the electron bunch energy with an optical laser before the bunch enters the radiator undulator. The resonant FEL wavelength is correlated to this energy modulation, and it may be possible to selectively filter [6] or amplify [7] a narrow wavelength band to generate short pulses with widths of a fraction of the modulation period. Other techniques rely upon similar electron bunch energy modulation methods [8,9]. Another approach, called E-SASE [10,11], uses an optical laser to modulate the electron bunch energy at an intermediate acceleration stage. Regions of enhanced current are created that subsequently generate short pulses in a final radiator undulator. A conceptually simpler method has also been suggested [12] that "spoils" all but a short region of the electron bunch. Only this region subsequently lases in the FEL to generate a short radiation pulse. More recently, a technique using an FEL with a negative undulator taper and a premodulated bunch energy has been proposed [13]. The above techniques typically predict pulse widths $\gtrsim 100$ as at a target wavelength of $\sim 1.5 \AA$.

Here, a technique is presented that may shorten the duration of $x$-ray pulses generated by SASE FELs to less than the atomic unit of time (24 as). By applying concepts from mode-locked cavity lasers [14], we predict generation of a train of multi-GW peak power pulses, at wavelength $1.5 \AA$, of width $\approx 23$ as with 150 as separation, and a power contrast ratio of approximately 60 . Such pulses may have sufficient power, spatial, and temporal resolution to offer a new scientific tool for observing the dynamics of atomicscale phenomena.

In a SASE FEL, shown schematically in Fig. 1(a), a relativistic electron beam propagates at mean velocity $v_{z}<c$ along an undulator and exponentially amplifies the initial spontaneous emission via the FEL instability [3]. Radiation emission is at the resonant FEL wavelength $\lambda_{r}=$ $\lambda_{u}\left(1+\bar{a}_{u}^{2}\right) / 2 \gamma_{0}^{2}$ where $\lambda_{u}$ is the undulator period, $\bar{a}_{u}$ is the rms undulator parameter, and $\gamma_{0}$ is the mean electron bunch energy in units of the electron rest mass energy. For exponential growth the undulator must be longer than the gain length of the FEL interaction $l_{g}=\lambda_{u} / 4 \pi \rho$ [3], where

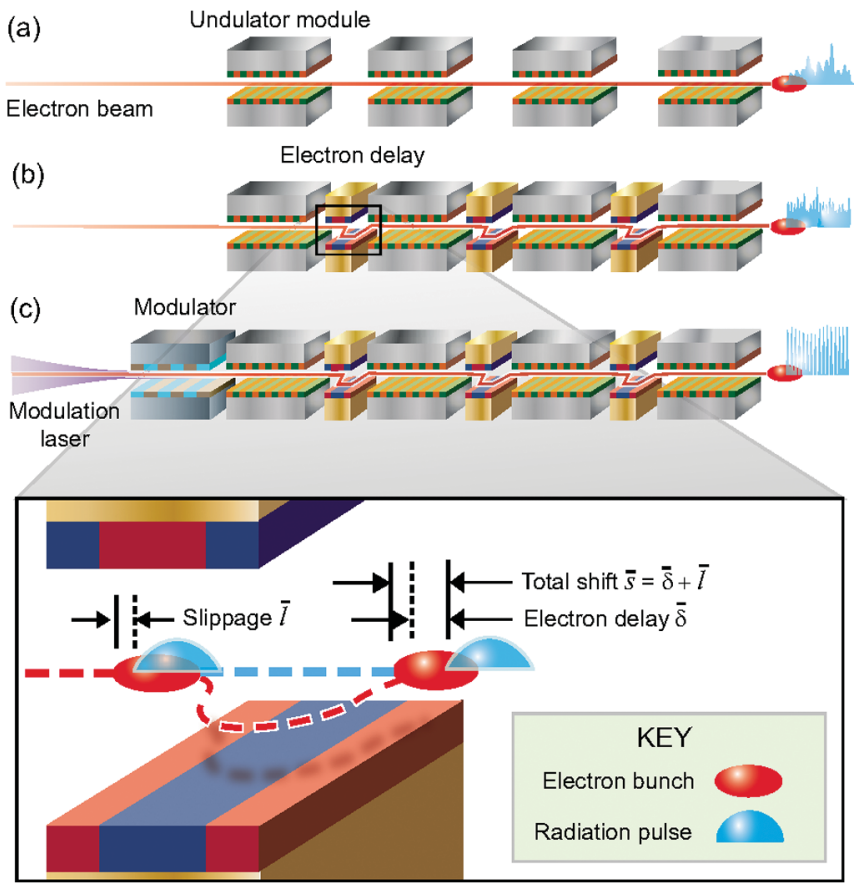

FIG. 1 (color). Schematic of three regimes of FEL interaction: (a) SASE regime (b) Mode-coupled SASE regime and (c) Modelocked SASE regime. The inset shows a detail of the electron delay. 
$\rho$ is the dimensionless FEL parameter, a measure of the coupling strength between electron beam and radiation, having typical values $\rho \sim 10^{-3}$ to $10^{-2}$.

The spatiotemporal range of the SASE interaction is different from a conventional laser: in the latter a radiation wave front interacts with the full length of the gain medium, whereas in a FEL a wave front may propagate through only a fraction of a typical electron bunch as $v_{z} \lesssim$ $c$. Autonomous regions of the radiation-electron interaction evolve from noise and are uncorrelated in phase. The temporal output power is noisy, comprising phaseuncorrelated pulses with separation $\leqslant 2 \pi l_{c}$, where the cooperation length $l_{c}=\lambda_{r} / 4 \pi \rho$ [15] is the relative propagation between a radiation wave front and electron bunch in one gain length and defines the intrinsic coherence length of the radiation $l_{\text {coh }} \approx l_{c}$. The SASE spectrum at saturation is similarly noisy comprising irregular spikes within rms bandwidth $\sigma_{\lambda} / \lambda_{r} \approx \rho$.

Here, techniques similar to those used in mode-locked broad bandwidth cavity lasers are used to improve the quality of the FEL output over SASE. The method generates a set of axial radiation modes, analogous to the axial cavity modes in conventional laser oscillators [14] which may be (actively or passively) phase locked by introducing a modulation with frequency $\Delta \omega_{s}$ equal to the mode spacing. Each mode acquires sidebands that overlap neighboring modes. The mode phases lock to generate temporal pulses spaced at, and with widths significantly shorter than, the cavity transit time [14].

The axial modes in the SASE FEL amplifier are synthesized by applying a series of spatiotemporal shifts between the radiation and copropagating electron bunch. These shifts are achieved by repeatedly delaying the electron bunch using magnetic chicanes between undulator modules, as shown schematically in Fig. 1(b). This "slippage" of the electron bunch behind the radiation is in addition to the slippage occurring within the undulator modules and increases the spatiotemporal coupling range over that of the simple SASE case, increasing both the cooperation and coherence lengths of the interaction. For equal, periodic delays, the radiation spectrum develops discrete frequencies similar to the axial modes of a conventional laser. These modes are intrinsically coupled via their collective interaction with the electron bunch over regions of a coherence length $l_{\text {coh }}$ within which a series of regularly spaced radiation spikes of approximately uniform width $\ll l_{\text {coh }}$ may evolve. Such a system, illustrated in Fig. 1(b), is termed "mode-coupled." By introducing an interaction modulation at the mode frequency spacing $\Delta \omega_{s}$, for example, a coherent modulation of the input electron bunch energy shown schematically in Fig. 1(c), the modes may become phase locked to give a temporal train of equally spaced, short, high-power pulses phase correlated over a distance $\gg l_{\text {coh }}$. The system is then termed "modelocked."
The effect of the spatiotemporal shifts on the radiation spectrum may be seen by considering the radiation emitted from a small, constant electron source term. In the universal scaling of $[3,15]$ the one-dimensional wave equation describing the field evolution may be written

$$
\frac{\partial A\left(\bar{z}, \bar{z}_{1}\right)}{\partial \bar{z}}+\frac{\partial A\left(\bar{z}, \bar{z}_{1}\right)}{\partial \bar{z}_{1}}=b_{0}\left(\bar{z}_{1}\right)
$$

where $A$ is the scaled electric field, $\bar{z}$ is the interaction length along the undulator in units of $l_{g}, \bar{z}_{1}$ is the scaled electron bunch coordinate in units of $l_{c}$, and the small initial electron source term $b_{0} \ll 1$. A solution to Eq. (1) for a single undulator and chicane module may be obtained using a Fourier transform with respect to $\bar{z}_{1}$ with no initial radiation $\tilde{A}(0, \bar{\omega})=0$. For a series of $N$ undulator and chicane modules a solution may be obtained by application of the Fourier transform time-shifting relation to yield

$$
\tilde{A}(\bar{L}, \bar{\omega})=\tilde{b}_{0} \bar{l} \operatorname{sinc}(\bar{\omega} \bar{l} / 2) \frac{e^{i N \bar{\omega} \bar{s}}-1}{e^{i \bar{\omega} \bar{s}}-1} e^{-i \bar{\omega} \bar{l} / 2},
$$

where the scaled interaction length $\bar{L}=N \bar{l}$, the total slippage per module in units of $\bar{z}_{1}$ is $\bar{s}=\bar{l}+\bar{\delta}, \bar{l}$ is the slippage due to the undulator, $\bar{\delta}$ is the slippage due to the chicane, the transform variable $\bar{\omega}=\left(\omega-\omega_{r}\right) / 2 \rho \omega_{r}$, and $\omega_{r}$ is the resonant FEL frequency. The expression (2) is identical in form to the result of a simple analysis that gives the axial mode content of a conventional cavity laser [14].

It is useful to define the slippage enhancement factor $S_{e}=\bar{s} / \bar{l}$. The extra slippage increases both $l_{c}$ and $l_{\text {coh }}$ by the factor $S_{e}$. For $S_{e}=1$ there are no chicane sections $(\bar{\delta}=0)$ and (2) reduces to the spontaneous emission spectrum for an undulator of scaled length $\bar{L}=N \bar{l}$. For $S_{e}>1$ the sinc-function envelope (the single undulator module spectrum) is modulated by a "frequency comb" centered at the scaled resonant frequency $\bar{\omega}=0$, with mode separation $\Delta \bar{\omega}=2 \pi / \bar{s}$ corresponding to a $\Delta \omega=2 \pi / T_{s}$ where $T_{s}=s / c=\bar{s} l_{c} / c$ is the time taken for radiation to travel the slippage length. In effect, the spectrum is that of a ring cavity of length $s$. Each sideband mode has width $\delta \bar{\omega} \propto\left(S_{e} N\right)^{-1}$. The $k$ th sideband at frequencies $\bar{\omega}_{k}=$ $\pm k \Delta \bar{\omega}$ will fall under the central peak of the sinc function when $S_{e}>k$. For the case $N=2$, Eq. (2) reduces to the spectrum of an optical klystron (OK) and agrees well with measured OK spectra $[16,17]$.

The delay chicanes may also introduce longitudinal energy dispersion parametrized via $D=R_{56} / 2 l_{c}[18,19]$, which for $D>0$ enhances the FEL gain process so reducing $l_{g}$ and $l_{c}$ by a nominal factor $G_{e} \leq 1$. Such enhanced gain is the principle behind the distributed optical klystron FEL amplifier [20-22]. The combined effects of the enhanced slippage $\left(S_{e} \geq 1\right)$ and gain length reduction $\left(G_{e}<\right.$ 1) give a modified cooperation length $\hat{l}_{c}=S_{e} G_{e} l_{c}$.

As illustrations of the technique two systems were simulated using the 3D code GENESIS 1.3 [23], used in the design of major facilities such as LCLS [24] and XFEL 
[25]. Parameters are given in Table I. The first system is a typical extreme ultraviolet (XUV) FEL design for operation at $124 \AA$. The radiation power output close to saturation over a $100 \mathrm{fs}$ sample for the three cases of SASE, mode coupling and mode locking of Figs. 1(a)-1(c), respectively, are shown in Fig. 2. Insets plot the power spectral density as a function of radiation wavelength. For the SASE case the output is seen to be noisy, comprising a series of irregularly spaced pulses with mean separation approximately $2 \pi l_{c}$, corresponding to a temporal duration $2 \pi \tau_{\text {coh }}=\lambda_{r} / 2 c \rho \approx 8 \mathrm{fs}$, with a spectrum centered at the resonant wavelength of fractional width $\approx 2 \rho$, results typical of the SASE regime [4].

In Fig. 2(b), the mode-coupled case, chicanes are introduced to give a delay $\delta=48 \lambda_{r}$, with total slippage due to undulator and delay section $s=60 \lambda_{r}$, a total slippage time of $T_{s} \approx 2.48 \mathrm{fs}$, slippage enhancement $S_{e}=5$, and gain length reduction factor $G_{e} \approx 0.65$. The spectrum has sideband modes with separation $\Delta \omega_{s}=2 \pi / T_{s}$, giving wavelength separation $\Delta \lambda \approx \lambda_{r}^{2} / s \approx 0.21 \mathrm{~nm}$. The power comprises a series of spikes with width $\tau_{p} \simeq 1 \mathrm{fs}$ FWHM and separation $T_{s} \approx 2.48 \mathrm{fs}$. The mode spiking can only be phase-matched over $\tau_{\text {coh }}=\hat{l}_{c} / c \approx 4.3$ fs. This results in the phase drifting of the spikes observed over the spike envelope.

When the beam energy is modulated at the mode separation frequency $\Delta \omega_{s}$, as shown schematically in Fig. 1(c), the radiation modes may phase lock over the entire bunch. A modulation amplitude of $5.8 \mathrm{MeV}$ was induced by seeding a 10 period modulator undulator, $\lambda_{u}=75 \mathrm{~mm}$, by a $230 \mathrm{MW} 755 \mathrm{~nm}$ laser. From Fig. 2(c) it is seen that mode-locking occurs, dramatically improving the temporal pulse structure over the SASE and mode-coupled cases of Figs. 2(a) and 2(b) to give a train of pulses, evenly spaced by $T_{s} \approx 2.48 \mathrm{fs}$, of constant width $\tau_{p} \approx 400$ as and peak power $\$ 1.4 \mathrm{GW}$. The contrast ratio is $>100$. The only remnant of the SASE noise is the slowly varying envelope of mean period $2 \pi \tau_{\text {coh }} \approx 27 \mathrm{fs}$.

TABLE I. XUV and $\mathrm{x}$-ray simulation parameters.

\begin{tabular}{lcc}
\hline \hline & XUV & X-ray \\
\hline Bunch energy $E(\mathrm{GeV})$ & 0.75 & 14.3 \\
Bunch peak current $I(\mathrm{kA})$ & 3 & 3.4 \\
Normalized emittance $\epsilon_{n}(\mathrm{~mm}-\mathrm{mrad})$ & 2 & 1.2 \\
RMS fractional energy spread $\sigma_{\gamma} / \gamma_{0}$ & $10^{-4}$ & $8 \times 10^{-5}$ \\
Undulator period $\lambda_{u}(\mathrm{~cm})$ & 3.1 & 3 \\
Resonant wavelength $\lambda_{r}(\AA)$ & 124 & 1.5 \\
Undulator module length $\left(\mathrm{units} l / \lambda_{u}\right)$ & 12 & 72 \\
FEL parameter $\rho$ & $2.5 \times 10^{-3}$ & $5 \times 10^{-4}$ \\
Chicane delay $N_{c}=\delta / \lambda_{r}$ & 48 & 228 \\
Modulation period (units of $\left.\lambda_{r}\right)$ & 61 & 303 \\
Modulation amplitude $(\mathrm{MeV})$ & 5.8 & 14.3 \\
Slippage enhancement $S_{e}$ & 5 & 5 \\
\hline \hline
\end{tabular}

The second system demonstrates the FEL mode-locking technique scaled to shorter wavelengths. Figure 3 plots the simulated saturated output power in the x-ray at $1.5 \AA$ for $S_{e}=5$ and other parameters similar to LCLS [24]. The pulse train consists of $\approx 23$ as pulses separated by $T_{s} \approx$ 150 as with peak powers up to $6 \mathrm{GW}$ and a contrast ratio $\approx 60$.

The output pulse width of a homogeneously broadened mode-locked cavity laser is given by [14] $\tau_{p} \approx$ $0.5 / \sqrt{N_{0}} f_{m}$ where $N_{0}$ is the number of oscillating modes in the cavity and $f_{m}$ the modulation frequency. Following the discussion of Eq. (2), the number of modes under the central peak of the spectrum is $N_{0}=2 S_{e}-1=9$, in good agreement with Figs. 2 and 3 where $N_{0} \approx 8$ in the XUV and $N_{0} \approx 9$ in the $\mathrm{x}$-ray. The previous expression then gives pulse lengths $\tau_{p} \approx 440$ as in the XUV and $\tau_{p} \approx$ 25 as in the $\mathrm{x}$-ray, in good agreement with the simulated values of $\tau_{p} \approx 400$ as and $\tau_{p} \approx 23$ as, respectively, further strengthening the analogy with the results of conventional mode-locked cavity lasers and indicating that the modes are phase locked.

We stress that the mode-locking technique described in this Letter does not rely upon the dispersive properties of the chicanes on the electrons - what is important is the relative electron bunch or radiation delay that creates the longitudinal modes. The technique works equally well for isochronous chicanes with $D=0$. Contrarily, the optical klystron effect relies only upon the chicane dispersion, requiring $D>0$, and does not rely upon any relative electron bunch or radiation delay. Furthermore, the role of the initial beam energy modulation in the mode-locking technique is to allow coupling and phase-locking of the synthesized longitudinal modes, whereas in the techniques
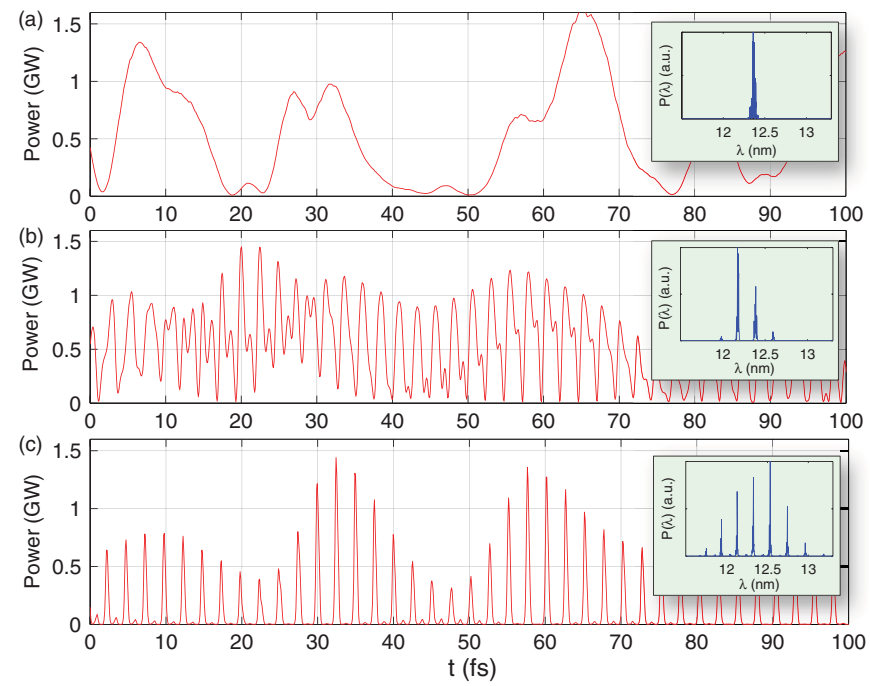

FIG. 2 (color). The radiation power as a function of time $t$ with radiation power spectrum as a function of wavelength in $\mathrm{nm}$ (inset) for the three cases of (a) SASE, (b) mode coupled, and (c) mode locked. 


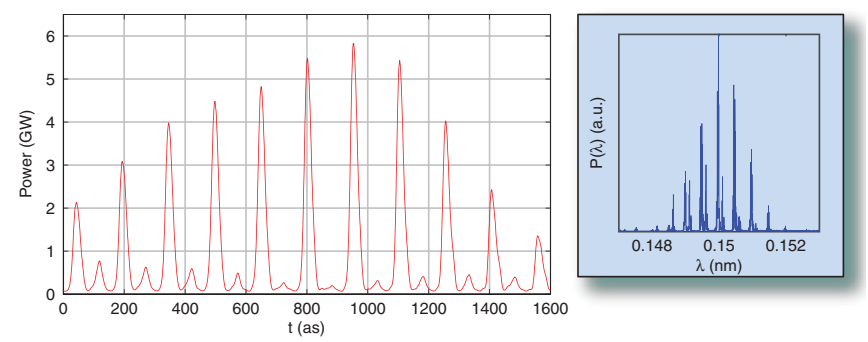

FIG. 3 (color). A mode-locked $1.5 \AA$ x-ray FEL example: the radiation power as a function of time $t$ with radiation power spectrum as a function of wavelength in $\mathrm{nm}$.

of [5-13], the beam energy modulation is primarily to allow either dispersive electron beam manipulation or to introduce energy chirp effects before radiation generation in the final FEL undulator sections.

A criterion for operation relates the electron beam energy spread to the dispersive strength of the chicane [18]: $\bar{\sigma}_{\gamma} \equiv \sigma_{\gamma} / \rho \gamma_{0} \lesssim 1 / D$. Approximating $R_{56} \simeq 10 \delta / 3$ gives $\sigma_{\gamma} / \gamma_{0} \lesssim 1 / 20 N_{c}$ where $N_{c}=\delta / \lambda_{r}$. The effect of magnet stability of the chicanes upon the electron delay $\delta$ must also be considered. For a 4-dipole chicane with equal magnet and drift lengths $L$, the delay may be written $\delta=$ $L \phi^{2}$, where $\phi$ is the small deflection angle. In a single dipole of field strength $B, \phi=e B L / \gamma_{0} m c$ so that $\Delta \delta=$ $2 \delta \Delta B / B$. Applying the requirement for subwavelength phase matching $\Delta \delta<\lambda_{r}$, and assuming that for $N$ independently powered chicanes the errors add as in a random walk, the tolerance on the magnetic field is then $\Delta B / B<$ $1 / 2 N_{c} \sqrt{N}$. The effect of shot-to-shot electron bunch energy fluctuation is also considered. From the chicane delay $\delta=L \phi^{2}$, and using the FEL resonance condition, the delay may be expressed as $\delta / \lambda_{r}=2 e^{2} B^{2} L^{3} / m^{2} c^{2} \lambda_{u}(1+$ $\bar{a}_{u}^{2}$ ) which is independent of the beam energy. Thus, beam energy fluctuations should not affect the axial mode structure as the chicanes always delay by the same number of resonant wavelengths. The numerical values predicted by these tolerance criteria for the XUV (and x-ray) systems are as follows: $\sigma_{\gamma} / \gamma_{0}<1 \times 10^{-3}\left(2 \times 10^{-4}\right)$ and $\Delta B / B<2 \times 10^{-3}\left(4 \times 10^{-4}\right)$ demonstrating that both systems are feasible with current technology.

The introduction of mode locking in conventional cavity lasers dramatically increased the range of their scientific application. It has been shown here that the use of similar techniques in FELs yields a similar leap in capability for what are already recognized as important tools for scientific investigation. Further advances may well be possible: alternative methods of locking the coupled modes or generating more exotic modal structures may enable pulse shaping in the attosecond domain; variation of the pulse structure may be achievable by using different chicane delays and tapered undulator sections.
The authors would like to thank Steve Jamison and Brian Sheehy for helpful discussions.

*n.r.thompson@dl.ac.uk

b.w.j.mcneil@strath.ac.uk

[1] P. B. Corkum and F. Krausz, Nature Phys. 3, 381 (2007).

[2] W. B. Colson, J. Blau, and A. Kampouridis, Proceedings of 28th International FEL Conference, THPPH071 (BESSY, Berlin, 2006), p. 756.

[3] R. Bonifacio, C. Pellegrini, and L. Narducci, Opt. Commun. 50, 373 (1984).

[4] R. Bonifacio et al., Phys. Rev. Lett. 73, 70 (1994).

[5] E. L. Saldin, E. A. Schneidmiller, and M. V. Yurkov, Opt. Commun. 212, 377 (2002).

[6] E. L. Saldin, E. A. Schneidmiller, and M. V. Yurkov, Opt. Commun. 237, 153 (2004).

[7] E. L. Saldin, E. A. Schneidmiller, and M. V. Yurkov, Opt. Commun. 239, 161 (2004).

[8] A. A. Zholents and W. M. Fawley, Phys. Rev. Lett. 92, 224801 (2004).

[9] A. A. Zholents and G. Penn, Phys. Rev. ST Accel. Beams 8, 050704 (2005).

[10] A. A. Zholents et al., Proceedings of the 2004 FEL Conference, Trieste, http://www.jacow.org/, pp. 582-585.

[11] Alexander A. Zholents, Phys. Rev. ST Accel. Beams 8, 040701 (2005)

[12] P. Emma, Z. Huang, and M. Borland, Proceedings of the 2004 FEL Conference, Trieste, http://www.jacow.org/, pp. 333-338.

[13] E. L. Saldin, E. A. Schneidmiller, and M. V. Yurkov, Phys. Rev. ST Accel. Beams 9, 050702 (2006).

[14] A.E. Siegman, Lasers (University Science Books, Sausalito, USA, 1986). See Chap. 27.

[15] R. Bonifacio, B. W. J. McNeil, and P. Pierini, Phys. Rev. A 40, 4467 (1989).

[16] R. Prazeres et al., Rev. Sci. Instrum. 60, 1429 (1989).

[17] R. Walker et al., Proceedings of 2000 European Particle Accelerator Conference, Vienna, http://www.jacow.org, p. 93.

[18] R. Bonifacio, R. Corsini, and P. Pierini, Phys. Rev. A 45, 4091 (1992).

[19] T. O. Raubenheimer, P. Emma, and S. Kheifets, Particle Accelerator Conference, Washington, DC, 1993, http:// www.jacow.org.

[20] V. N. Litvinenko, Nucl. Instrum. Methods Phys. Res., Sect. A 304, 463 (1991).

[21] G. R. Neil and H. P. Freund, Nucl. Instrum. Methods Phys. Res., Sect. A 475, 381 (2001).

[22] Y. Ding et al., Phys. Rev. ST Accel. Beams 9, 070702 (2006).

[23] S. Reiche, Nucl. Instrum. Methods Phys. Res., Sect. A 429, 243 (1999).

[24] J. Arthur et al., SLAC Report No. SLACR593, 2002.

[25] M. Altarelli et al., "The European X-Ray Free-Electron Laser Technical Design Report," Deutsches ElektronenSynchrotron, 2007. 